\title{
Systemic inflammation and chronic kidney disease in a patient due to the RNASEH2B defect
}

Tingyan $\mathrm{He}^{*}$, Yu Xia and Jun Yang ${ }^{*}$

\begin{abstract}
Introduction: Aicardi-Goutières (AGS) is a rare immune dysregulated disease due to mutations in TREX1, RNASEH2A, RNASEH2B, RNASEH2C, SAMHD1, ADAR1, or IFIH1. Clinical features include basal ganglia calcifications, white matter abnormalities, and cerebral atrophy. Severe systemic inflammation and chronic kidney disease (CKD) are extremely rare in AGS. Herein, we report a patient presenting with systemic inflammation and CKD to broaden the clinical phenotype spectrum of the RNASEH2B defect.

Methods: All testing and molecular genetic analysis were performed after obtaining the informed consent of the parents. Demographic, clinical, and laboratory findings were abstracted from outpatient and inpatient encounters. Cerebral magnetic resonance imaging (MRI), computed tomography (CT) scans, and renal biopsy histopathology reports were reviewed and summarized. Whole exome sequencing (WES) was performed on peripheral blood cells. After exposure to CGAMP in vitro for $24 \mathrm{~h}$, mRNA expression of 12 IFN-stimulated cytokine genes in PBMCs was assessed. Serum cytokine levels were detected by Milliplex.
\end{abstract}

Results: A 11-year-old girl presented with recurrent aseptic fever, arthritis, chilblains, failure to thrive, mild hearing loss, and neurological manifestations. Laboratory and immunologic findings demonstrated lymphopenia, low complement levels, positive autoantibodies, elevated levels of acute-phase reactants and inflammatory cytokines. Cerebral imaging showed cerebral atrophy, white matter abnormalities, and intracranial calcification. Renal biopsy showed glomerular sclerosis in 3 of 14 glomeruli, infiltration of lymphocytes and other mononuclear cells. WES revealed a homozygous and heterozygous mutations in RNASEH2B. Over-expression of IFN-stimulated cytokine genes was observed, including IFI44, IFI27, IFIT1, IFIT2, IFIT3, ISG15, OAS1, and SIGLEC1.

Conclusions: To date, only two cases with AGS have been reported to have renal disease. Here, we describe a patient with both homozygous and heterozygous variants in RNASEH2B, presenting with neurological manifestations, persistently systemic autoinflammation, and CKD. CKD has never been reported in patients with AGS due to the RNASEH2B defect.

Trial registration: Not applicable; this was a retrospective study.

Keywords: Auto-inflammation, Autoimmunity, Aicardi-Goutieres syndrome, Chronic kidney disease, RNASEH2B

\footnotetext{
*Correspondence: hetingyan2017@outlook.com; rogasansz@163.com Department of Rheumatology and Immunology, Shenzhen Children's Hospital, 7019 Yitian Road, Shenzhen 518038, China
} 


\section{Background}

Aicardi-Goutières (AGS) is a rare immune dysregulated disease due to mutations in TREX1, RNASEH2A, RNAS EH2B, RNASEH2C, SAMHD1, ADAR1 or IFIH1, characterized by encephalopathy, dystonia, basal ganglia calcifications, white matter abnormalities, and cerebral atrophy [1, 2]. Although most patients experienced severe neurological dysfunction within the first year of life, some patients presented with later onset of this disease with mild neurological manifestations and normal intellectual function. Systemic inflammation is not typically persistent. Renal dysfunction has been rarely described in AGS [2]. Here, we report a patient with both homozygous and heterozygous mutations in RNAS $E H 2 B$, presenting with later onset recurrent sterile fever, arthritis, chilblains, failure to thrive, mild hearing loss, and neurological manifestations, which may broaden the clinical phenotype spectrum of the RNASEH $2 B$ defect.

\section{Materials and methods Subjects}

This study was approved by the Ethics Committee of Shenzhen Children's hospital. All human subjects (or their guardians) provided written informed consent. Clinical data of a patient with both homozygous and heterozygous variants in RNASEH $2 B$ was collected. Fifteen healthy volunteers were included as healthy controls (HCs). Venous blood $(3 \mathrm{~mL})$ was collected from each study subject.

\section{Whole exome sequencing (WES)}

Genomic DNA was extracted from peripheral blood cells isolated from the patient and her parent. The exonic regions and flanking splicing or intronic junctions of the whole genome were captured and sequenced using an Illumina HiSeq 2000 sequencer conducted by MyGenostics (Beijing, China). The FASTQ files were mapped to the human reference genome (hg19). The functional effects of variants were predicted using three algorithms (PolyPhen-2, SIFT, and MutationTaster), and amino acid conservation among species was analyzed. Sanger sequencing was used to confirm pathogenic variants. The primers used to target human RNASEH2B included (forward: CAGGGATTTGAAGCTCTTTGG) and (reverse: TAGTGCTCTGTCCTGCACTGG).

\section{Cell culture}

Peripheral blood mononuclear cells (PBMCs) were isolated by Ficoll-Paque PLUS (GE Healthcare) gradient density centrifugation and ACK lysis (Quality Biological). PBMCs were resuspended in complete RPMI (cRPMI) medium (Gibco, USA) containing 10\% fetal bovine serum (BI, Israel), $2 \mathrm{mM}$ glutamine, and penicillinstreptomycin (100 U/mL each; Sigma-Aldrich, USA).
Cells at $1 \times 10^{6} / \mathrm{mL}$ were exposed to cyclic guanosine monophosphate-adenosine monophosphate (cGAMP, CST\#35573) at the concentration of $10 \mu \mathrm{g} / \mathrm{ml}$.

\section{Real-time PCR}

After exposure to cGAMP in vitro for $24 \mathrm{~h}$, mRNA expression of 12 IFN-stimulated cytokine genes in PBMCs was assessed. Total RNA was extracted from PBMCs isolated from the patient and five HCs by RNA isolation kit (DP424, TIANGEN). cDNA was derived following the GoScript Reverse Transcription System kit(A5001, Promega). Quantitative reverse transcription PCR analysis was performed with the GoTaq qPCR Master Mix (A6002, Promega). Primers for PCR included were described in the supplementary material.

\section{Quantification of cytokine levels}

Plasma samples were isolated from the patient and 15 HCs. Cerebrospinal fluid (CSF) sample was collected from the patient. Blood samples were collected in vacutainers containing sodium heparin. Plasma cytokine analyses were determined on a bead-based immunoassay (Milliplex, HCYTOMAG-60 K, Millipore, USA) according to the manufacturer's protocol.

\section{Statistical analysis}

Data were analyzed using an unpaired two-tailed Student t-test. All statistical analyses were conducted in GraphPad Prism 7 software (GraphPad Software, Inc., San Diego, CA).

\section{Results}

\section{Clinical manifestations}

The patient presented with recurrent fever, arthritis, movement limitation, and growth retardation at the age of 11 years. At the age of 2 years, she began to suffer from recurrent aseptic fever with an intermittent resolution by traditional Chinese medicine. At the age of 5 years, she began to present with arthritis accompanied by mild hearing loss. She was born to a nonconsanguineous healthy parent. At birth, her weight was $3 \mathrm{Kg}$, crown to heel length was $49 \mathrm{~cm}$, and head circumference was $34 \mathrm{~cm}$. She had standard motor and language development. Manifestations of failure to thrive had been significant since she was 3 years old. Physical examination revealed short stature with $106 \mathrm{~cm}$ top $(<$ -3SD)(Fig. 1B3), macrocephaly with $54 \mathrm{~cm}$ head width, chilblains on elbows and lower limbs (Fig. 1B2), swelling and deformation of inter-phalangeal and knee joints (Fig. 1B1). Her Intelligence Quotient (IQ) test value was 108. Her EPQJ, CBCL, Conners, and HAMA scale tests did not demonstrate any social and psychological problems. Knee magnetic resonance imaging (MRI) revealed a thickness of the synovial capsule without invasive 


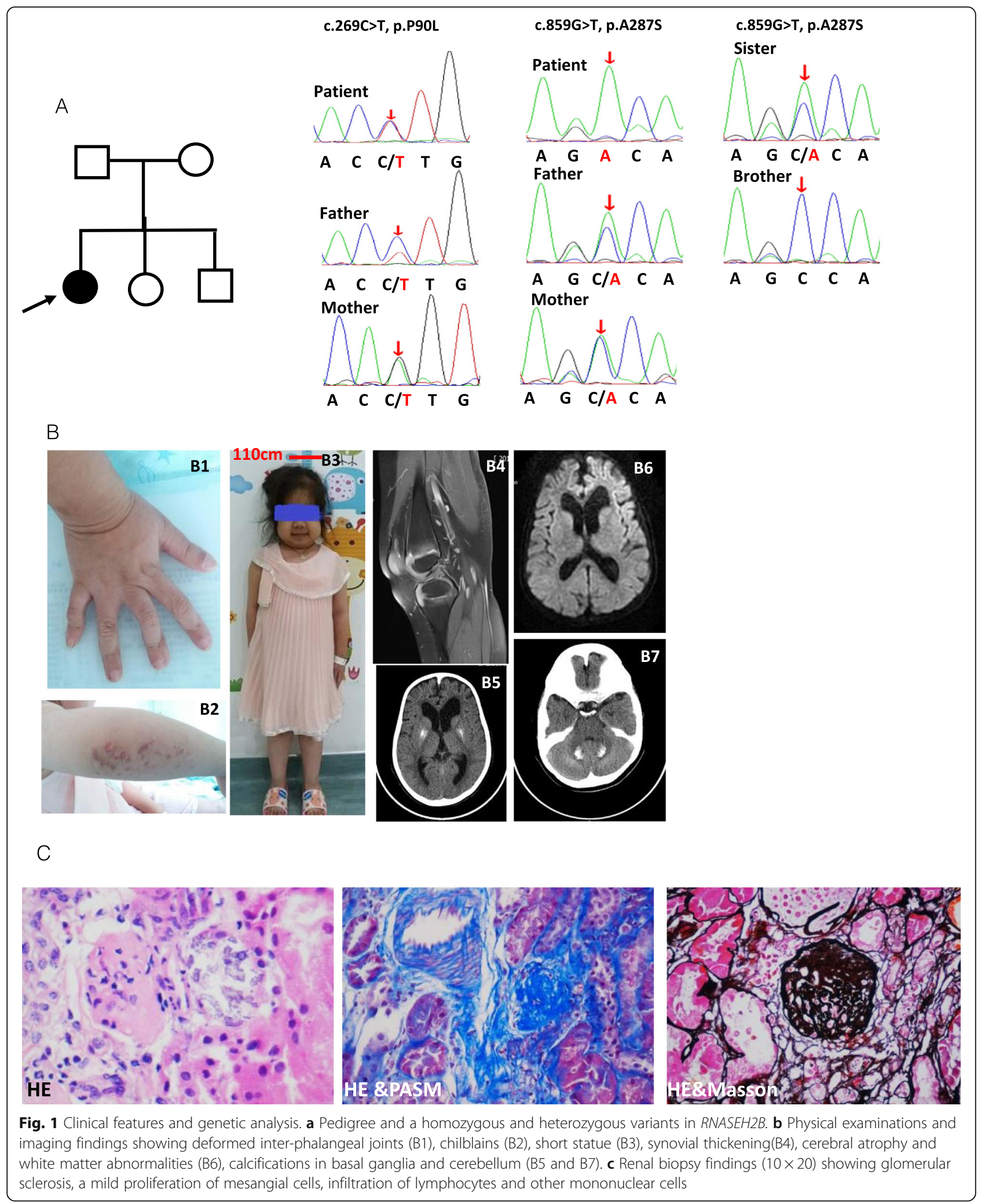

bone destruction (Fig. 1B4). Cerebral MRI showed cerebral atrophy and white matter abnormalities (Fig. 1B6). Intracranial calcification was further identified at the basal ganglia and cerebellum by CT scanning (Fig. 1B5 and Fig. 1B7). Laboratory findings revealed hyper-inflammation and chronic kidney 
disease (Fig. 1c, Fig. 2b, and Fig. 2f). Screening tests for fungal, bacteria, and Mycobacterium tuberculosis infection were all negative. Pathology of the renal biopsy showed glomerular sclerosis in 3 of 14 glomeruli, a mild proliferation of mesangial cells without deposits of any amyloid, immunoglobulin or immune complex, expansion of the tubular lumen, partial tubular atrophy, mild tubular fibrosis, infiltration of lymphocytes and other mononuclear cells (Fig. 1c). Granule degeneration and calcium deposition were visible in renal tubules. Austin score index for the evaluation of activity and chronicity was two and three points, respectively.

\section{Abnormality in clinical and immunologic phenotype}

Analysis of peripheral blood leukocyte revealed persistent lymphopenia (Fig. 2a). Except for rheumatoid factor (RF) and anti-cyclic peptide containing citrulline (anti$\mathrm{CCP}$ ), other auto-antibodies for mixed connective tissue disease were all negative, including anti-nuclear antibody
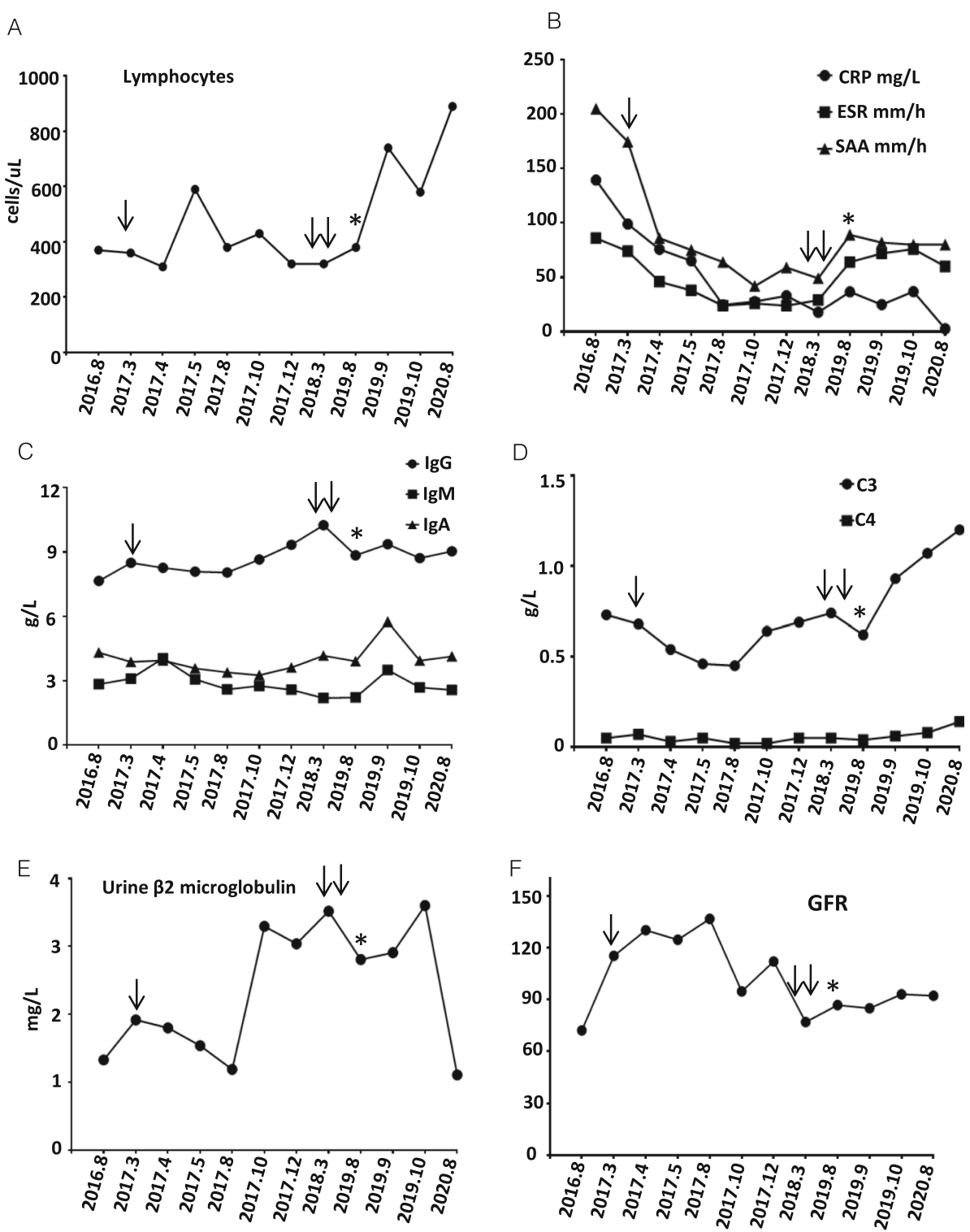

Fig. 2 Abnormalities in laboratory findings. a lymphopenia. b Elevated CRP, ESR, and SAA levels. c Immunoglobulin levels showing intermediate elevated IgM and IgA levels. $\mathbf{d}$ Reduced C3 and C4 levels. e Persistently elevated levels of urine $\beta 2$ macroglobulin. $\mathbf{f}$ Intermediate mild abnormalities in $\mathrm{Cr}$ and BUN. The normal reference ranges are as follows: lymphocytes (800 $4000 \mathrm{cells} / \mathrm{ul})$; CRP $(0 \sim 10 \mathrm{mg} / \mathrm{L})$; ESR $(0 \sim 20 \mathrm{~mm} / \mathrm{h})$; SAA $(0 \sim 6 \mathrm{mg} / \mathrm{L}) ; \operatorname{lgG}(5.28 \sim 21 \mathrm{~g} / \mathrm{L})$; IgM $(0.48 \sim 2.26 \mathrm{~g} / \mathrm{L}) ; \operatorname{lgA}(0.44 \sim 3.99 \mathrm{~g} / \mathrm{L}) ; C 3(0.7 \sim 2.06 \mathrm{~g} / \mathrm{L}) ; C 4$ (0.11 0.61 g/L); urine $\beta 2$ macroglobulin $(0$ $\sim 0.3 \mathrm{mg} / \mathrm{L})$; GFR $(80 \sim 120 \mathrm{ml} / \mathrm{min}$ ). Single arrow and double arrows labeled the time when tocilizumab was started and discontinued, respectively. The asterisk labeled the time when tofacitinib was started 
(ANA), anti-neutrophil cytoplasmic antibody (ANCA), anti-SSA, anti-SSB, anti-dsDNA, anti-thyroglobulin, anti-thyroperoxidase, and anti-TSH receptor antibodies. Other abnormal clinical and immunologic phenotypes included intermediate elevation of $\operatorname{IgM}$ and IgA levels (Fig. 2c) and mild reduction of $\mathrm{C} 3$ and $\mathrm{C} 4$ levels (Fig. 2d).

\section{Both homozygous and heterozygous variants in RNAS EH2B}

Whole exon sequencing revealed three variants in the RNAS $E H 2 B$ gene (OMIM:610181). There was a single nucleotide homozygous variant, c.859G > T, p.A287S (Fig. 1a). Predicted values of SIFT, PolyPhen_2, Mutation Taster, and GERP++ were $0.235,0.721,1$, and 6.06 , suggesting tolerated, possibly damaging, and disease-causing effects, respectively. Both parents carried a heterozygous mutation at the same locus.
Another single-nucleotide heterozygous variant, c.269C > T, p.P90L, was identified (Fig. 1a). The predicted values of SIFT, PolyPhen_2, Mutation Taster, and GERP++ were 0.002, $0.988,1$, and 4.69 , suggesting damaging, probably damaging, and disease-causing effects, respectively. This heterozygous variation was further confirmed by Sanger in both her parents. Both variations were in the conserved domains. Pathogenic variants were not identified in other genes related to autoinflammation, autoimmunity, or inherited renal disorders (Supplemental Table).

\section{Over-expression of IFN-stimulated cytokine genes}

After exposure to cGAMP in vitro for $24 \mathrm{~h}, \mathrm{mRNA}$ expression of IFN-stimulated cytokine genes in PBMCs was detected by real-time PCR. In contrast to five healthy controls, over-expression of IFN-stimulated cytokine genes was observed in the patient, including
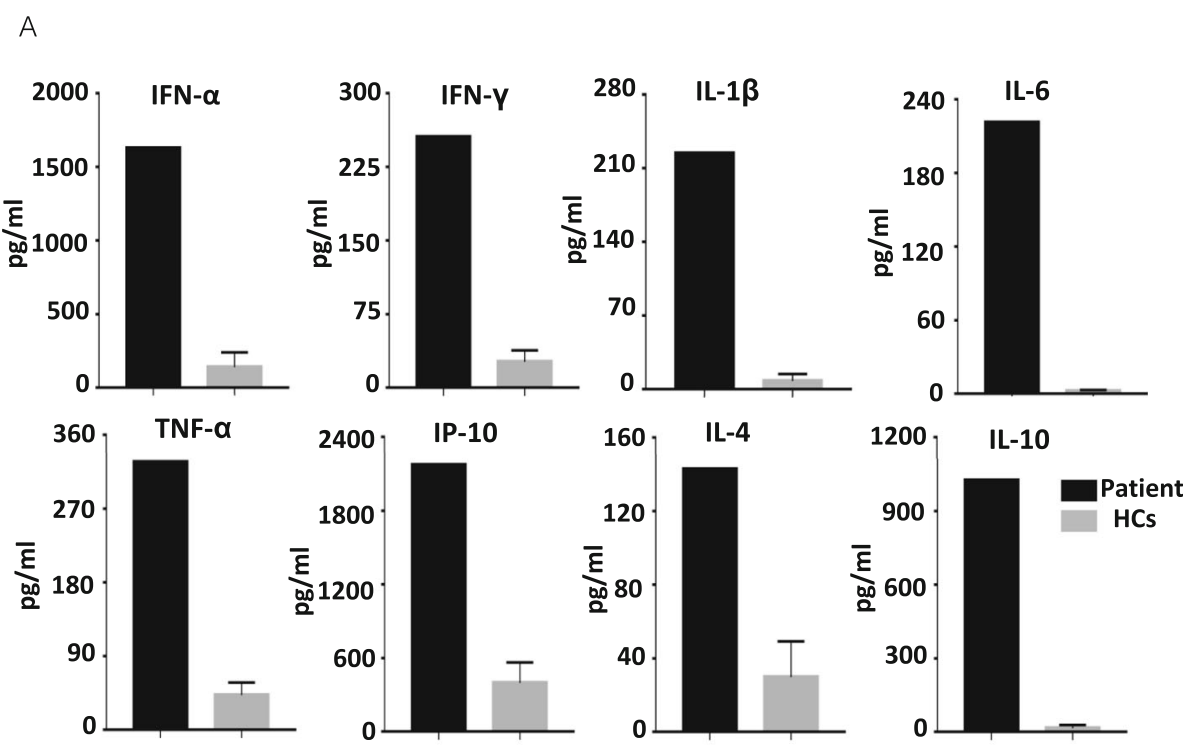

B

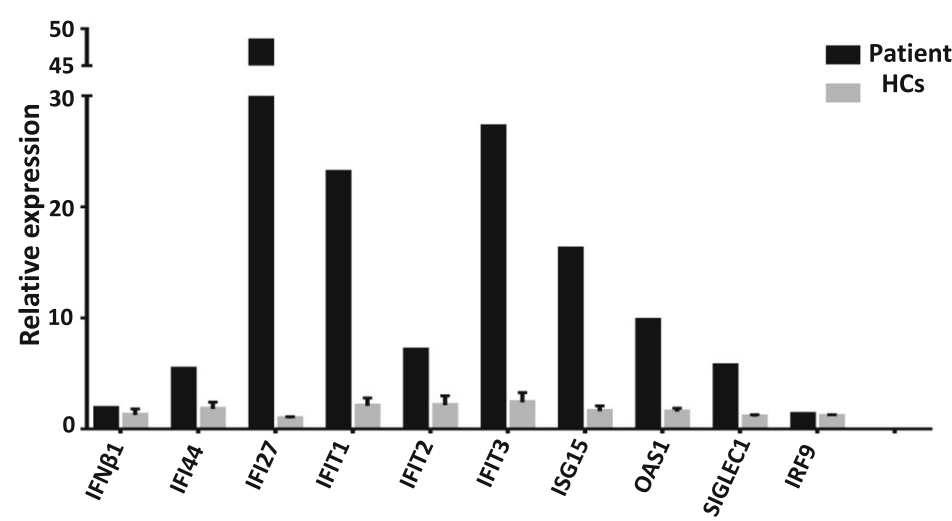

Fig. 3 Abnormalities in plasma cytokines and IFN-stimulated cytokine genes. a Significantly elevated levels of all plasma cytokines in the patient. b Over-expression of IFN-stimulated cytokine genes in the patient, including IFI44, IFI27, IFIT1, IFIT2, IFIT3, ISG15, OAS1, and SIGLEC1. Normal mRNA expressions were observed in IFN $\beta 1$ and IRF9 
IFI44, IFI27, IFIT1, IFIT2, IFIT3, ISG15, OAS1, and SIGLEC1. Normal mRNA expressions were found in IFN $\beta 1$ and IRF9 (Fig. 3b).

\section{Elevations in inflammatory cytokine levels} Compared to 15 age-matched healthy controls, plasma cytokine levels were significantly elevated, including interleukin (IL)- $1 \beta$, IL-6, tumor necrosis factor- $\alpha$ (TNF $\alpha)$, interferon- $\gamma$ (IFN- $\gamma$ ), IFN- $\alpha$, IL-4, IL-10, IL-12, IL-17A and IP-10 (Fig. 3a and Table 1). IFN- $\alpha$ level in CSF was very low.

\section{Treatment and outcome}

A one-year course of growth hormone showed no response to improve her short stature. She had received long-term treatment of ibuprofen, methotrexate, folic acid, and prednisone for more than 5 years. Aseptic fever relapsed intermittently. Tocilizumab was started for the high dose dependence of glucocorticoids and elevated pro-inflammatory cytokine levels. Following a 48week course of tocilizumab, the prednisone dose was gradually reduced to $0.20 .3 \mathrm{mg} / \mathrm{Kg} . \mathrm{d}$ with partial improvement of some abnormal laboratory findings (Fig. $2 \mathrm{~b}$ and Fig. 2f). However, urine $\beta 2$ microglobulin level was persistently elevated markedly (Fig. 2e). Tocilizumab was discontinued. She began to receive tofacitinib $(5 \mathrm{mg}$, twice every day) for the over-expression of IFN-stimulated cytokine genes. The 48-week course of tofacitinib led to partial response, including increased lymphocytes, C3 and C4 levels, reduced levels of urine $\beta 2$ microglobulin, $C$-reactive protein (CRP), and pro-inflammatory cytokines (Fig. 2a and Fig. 2d). However, chronic systemic inflammation was not completely controlled since the levels of inflammatory cytokines, erythrocyte sedimentation rate (ESR), and serum amyloid A (SAA) were still increased.

\section{Discussion}

Biallelic mutations of RNASEH $2 B$ are most common in AGS. While three allelic variants in RNASEH $2 B$ have been identified in this patient. The population frequency of the variant c.859G > T, p.A287S in East Asian is 0.02, with two homozygotes demonstrated in ExAC Browser. This variation can cause reduced enzyme activity of RNase $\mathrm{H} 2$ and lower stability of the RNase $\mathrm{H} 2$ complexes, increasing susceptibility to systemic lupus erythematosus (SLE) [3]. The heterozygous variant c. $269 \mathrm{C}>\mathrm{T}$, p.P90L is highly conserved with extremely low population frequencies and no homozygotes in ExAC Browser. Its clinical significance remains uncertain in ClinVar. The tri-allelic mutations in RNASEH $2 B$ may cause a synergistic pathogenic effect since neither heterozygous nor homozygous variants alone can account for her skin and neurological manifestations.

This patient has demonstrated a later onset of AGS with average intelligence, presenting with chilblains, cerebral atrophy, white matter abnormalities, intracranial calcification, and over-expression of Interferonstimulated genes. Besides, this patient has persistent systemic inflammation and chronic renal dysfunction, which are uncommon in AGS (Table 2). Mixed connective tissue diseases have been excluded by the systemic evaluation. Systemic juvenile idiopathic arthritis (SoJIA), and later onset chronic infantile neurologic, cutaneous, and arthritis (CINCA) syndrome were once suspected.

Table 1 Other laboratory findings

\begin{tabular}{|c|c|c|c|c|c|}
\hline Parameters & $\begin{array}{l}\text { Before } \\
\text { tocilizumab }\end{array}$ & $\begin{array}{l}12 \text { weeks after } \\
\text { tocilizumab }\end{array}$ & $\begin{array}{l}\text { Before } \\
\text { tofacitinib }\end{array}$ & $\begin{array}{l}48 \text { weeks after } \\
\text { tofacitinib }\end{array}$ & $\begin{array}{l}\text { Reference } \\
\text { Range }\end{array}$ \\
\hline Rheumatoid factor (IU/ml) & 879 & 810 & 2615 & 2660 & $0-20$ \\
\hline Protein in CSF (mg/L) & 541.9 & NA & NA & NA & $150-450$ \\
\hline WBCs in CSF $\left(10^{6} / \mathrm{L}\right)$ & 1 & NA & NA & NA & $0-15$ \\
\hline interferon-a in CSF (pg/ml) & 22.1 & NA & NA & NA & NA \\
\hline $\begin{array}{l}\text { interferon-a in plasma (pg/ } \\
\text { ml) }\end{array}$ & 1627 & 1040 & 2309 & 1601.4 & $139.64 \pm 96.54$ \\
\hline IP10 in plasma (pg/ml) & 2172 & 2160 & 3702 & 2135 & $397.43 \pm 159.51$ \\
\hline IFNy in plasma (pg/ml) & 453.6 & 260.8 & 850.5 & 282.9 & $26.32 \pm 11.24$ \\
\hline TNFa in plasma (pg/ml) & 335.4 & 232.9 & 554.6 & 259.1 & $42.05 \pm 14.76$ \\
\hline IL1 $\beta$ in plasma (pg/ml) & 224.15 & 208 & 274.3 & 218 & $7.75 \pm 6.5$ \\
\hline IL4 in plasma (pg/ml) & 311 & 135.6 & 473 & 252 & $29.81 \pm 18.48$ \\
\hline IL6 in plasma (pg/ml) & 221 & 142.6 & 230.3 & 156.2 & $5.5 \pm 3.39$ \\
\hline IL10 in plasma (pg/ml) & 1025 & 540.9 & 731.4 & 568.6 & $14.36 \pm 9.88$ \\
\hline IL12 in plasma (pg/ml) & 1507 & 1389 & 1372 & 1337 & $149.32 \pm 139.9$ \\
\hline IL17A in plasma (pg/ml) & 64.9 & 44.8 & 43.7 & 48 & $15.55 \pm 10.13$ \\
\hline GM-CSF in plasma (pg/ml) & 1146 & 1053 & 1067 & 1073.6 & $68.36 \pm 44.74$ \\
\hline
\end{tabular}


Table 2 Comparison of clinical features among AGS, soJIA, and CINCA

\begin{tabular}{|c|c|c|c|c|}
\hline Clinical features & Our Patient & Other AGS $[2,3]$ & soJlA [4-6] & CINCA $[7,8]$ \\
\hline Onset within the first year of life & no & common & rare & common \\
\hline Fever & frequently often & rare & common & common \\
\hline Preserved or normal intelligence & yes & less common & yes & less common \\
\hline Mental retardation & no & common & rare & common \\
\hline Joint swelling & yes & rare & common & common \\
\hline Arthralgia & yes & rare & common & common \\
\hline Destructive arthritis & no & rare & common & common \\
\hline Chilblains & yes & common & NR & rare \\
\hline Urticarial rash & no & rare & less common & common \\
\hline Salmon-pink rash & no & rare & common & less common \\
\hline Conjunctivitis & no & none & less common & common \\
\hline Visual damage & no & less common & rare & common \\
\hline Sensor neural deafness & no & rare & NR & common \\
\hline Progressive chronic meningitis & no & rare & NR & common \\
\hline Auto-inflammatory manifestations & yes & less common & common & common \\
\hline Auto-immune manifestations & no & common & rare & none \\
\hline Severe intra-uterine growth retardation & no & common & NR & rare \\
\hline Microcephaly & no & common & NR & rare \\
\hline Psychomotor retarded & not obvious & common & rare & common \\
\hline Feeding difficulties & no & common & NR & less common \\
\hline Growth retardation & yes & common & less common & common \\
\hline Hepatosplenomegaly & no & common & common & common \\
\hline Cerebral atrophy & milder & common & NR & common \\
\hline White matter abnormalities & yes & common & NR & less common \\
\hline Intracranial calcification & yes & common & NR & none \\
\hline Chronic kidney disease & yes & none & rare & common \\
\hline Renal amyloidosis & not yet & none & rare & common \\
\hline Leukocytosis & no & rare & common & common \\
\hline C-reactive protein & significantly elevated & rare & significantly elevated & significantly elevated \\
\hline Erythrocyte sedimentation & significantly elevated & less common & significantly elevated & significantly elevated \\
\hline Ferritin & normal & normal & significantly elevated in MAS & significantly elevated in MAS \\
\hline Triglycerides & normal & normal & elevated in MAS & elevated in MAS \\
\hline
\end{tabular}

AGS Aicardi-Goutières, SoJIA Systemic juvenile idiopathic arthritis, CINCA onset chronic infantile neurologic, cutaneous, and arthritis syndrome, NR not reported, MAS macrophage activation syndrome

Different from the clinical manifestations of this patient, chilblains and intracranial calcification are not present in SoJIA or CINCA; leukocytosis, destructive arthritis, or macrophage activation syndrome (MAS) are noted in SoJIA [4-6]; visual impairment, sensor neural deafness or progressive chronic meningitis have been commonly reported in CINCA [5]. Chronic kidney disease due to amyloidosis has been rarely reported in SoJIA, which is common in CINCA (Table 2) [8].

Renal involvement has been described in a case with a gain-of-function mutation in IFIH1 [9]. Renal dysfunction caused by thrombotic microangiopathy has been reported in a case with C-terminal frame-shift mutation in TREX1 [10]. Renal biopsy in our patient revealed glomerular sclerosis and tubular injury without amyloidosis. RNAS EH2B is moderately expressed in the kidney. Pathogenic variations in $R N A S E H 2 B$ might impair the normal function of kidney directly, or secondary to chronic inflammation. Human IFN-alpha is filtrated by the kidney, primarily reabsorbed, most probably catabolized within the tubular epithelium, and excreted in negligible amounts with the urine [11]. A fairly high IFN-a level within the tubular epithelium due to a persistently elevated IFN-a level in plasma might amplify the activation of the 
interferon pathway, leading to the infiltration of lymphocytes and mononuclear cells, and local chronic inflammation. Further investigations will help to explore the distinct pathogenesis underlying chronic renal dysfunction in the RNASEH $2 B$ defect.

IL-6 is one of the downstream effector cytokines in the IFN signaling pathway. IL-6 blockade has good efficacy in a patient with a cerebral vasculopathy due to a homozygous SAMHD1 mutation [12]. Tocilizumab has partial efficacy in this patient, leading to a reduction of acutephase reactants. However, it has failed to improve the chronic renal tubular disease. Further clinical trials are required to clarify the efficacy of tocilizumab in AGS.

IFN- $\alpha$ and IFN- $\beta$ act on type I receptors (IFNAR1/2) to activate the Janus kinase (JAK)-signal transducers and activators of the transcription (STAT) pathway. JAK inhibitors have good efficacy in patients with some type I interferonopathies, including STING-associated vasculopathy, infantile-onset (SAVI), and proteasomeassociated autoinflammatory syndrome (PRAAS) [13-16]. Sustained elevated IFN- $\alpha$ and IFN- $\beta$ levels are common in AGS. JAK inhibitors can theoretically help to reduce the autoinflammation in AGS. Ruxolitinib has reduced neuroinflammation in a patient with a heterozygous mutation in IFIH1 [17]. Baricitinib could alleviate chilblain lesions in a patient with AGS5 [18]. Tofacitinib ameliorated aortic valve calcification in a patient with Singleton-Merten syndrome (SMS) [19]. Ruxolitinib led to an improvement of psychomotor delay with a reduction in dystonic movements in two patients with AGS2 [20]. However, ruxolitinib failed to prevent the onset of clinical signs in a patient with RNASEH2B mutation [21]. Tofacitinib demonstrated a partial response in this patient, failing to ameliorate autoinflammation and chronic kidney disease completely. Therefore, based on limited case reports, the efficacy of JAK inhibitors in AGS remains uncertain. The currently ongoing trial conducted at the Children's Hospital of Philadelphia (ClinicalTrials.gov number, NCT03921554) will help to explore the efficacy and safety of baricitinib in AGS and AGS-related interferonopathies.

\section{Conclusions}

We have described a patient with both homozygous and heterozygous variants in $R N A S E H 2 B$, revealing a possible synergistic pathogenic effect among variants in the same gene. Her systemic autoinflammation and chronic kidney disease will expand the clinical phenotype spectrum of this syndrome. The pathogenesis underlying chronic renal dysfunction in this patient remains poorly understood. The efficacy of tocilizumab and JAK inhibitors in AGS remains uncertain, and further clinical researches are needed.

\section{Supplementary Information}

The online version contains supplementary material available at https://doi. org/10.1186/s12969-021-00497-2.

\section{Additional file 1.}

Additional file 2.

\section{Abbreviations}

AGS: Aicardi-Goutières; WES: Whole exome sequencing; PBMCs: Peripheral blood mononuclear cells; cGAMP: cyclic guanosine monophosphateadenosine monophosphate; IQ: Intelligence Quotient; MRI: Magnetic resonance imaging; RF: Rheumatoid factor; ANA: Anti-nuclear antibody; ANCA: Anti-neutrophil cytoplasmic antibody; TNFa: Tumor necrosis factor-a; IFN- $\gamma$ : Interferon- $\gamma_{;}$CRP: C-reactive protein; ESR: Erythrocyte sedimentation rate; SAA: Serum amyloid A; SLE: Systemic lupus erythematosus;

SoJIA: Systemic juvenile idiopathic arthritis; CINCA: Onset chronic infantile neurologic, cutaneous, and arthritis syndrome; MAS: Macrophage activation syndrome; JAK: Janus kinase; SAVI: STING-associated vasculopathy, infantileonset; PRAAS: Proteasome-associated autoinflammatory syndrome;

SMS: Singleton-Merten syndrome

\section{Acknowledgments}

The authors wish to thank all the patients, their families, and healthy controls for the participation.

\section{Authors' contributions}

Tingyan He performed the main experiments, analyzed the data, and drafted the manuscript. Yu Xia collected clinical data from the patient. Jun Yang reviewed the manuscript. All authors read and approved the final manuscript.

\section{Funding \\ This work was supported by the Sanming Project of Medicine in Shenzhen (SZSM201812002), Science and Technology Planning Project of Shenzhen Municipality (JCY20170303155201082), and Shenzhen Key Medical Discipline Construction Fund (SZGSP012).}

\section{Availability of data and materials}

Clinical datasets were collected from medical records of the participated patient in Shenzhen Children's hospital.

\section{Ethics approval and consent to participate}

All participated family members were enrolled upon approval of the ethics committee of Shenzhen Children's hospital and written consent of all the families.

\section{Consent for publication}

Written consent for publication of this anonymous information was obtained from the patient's parents.

\section{Competing interests}

All authors declare no conflict of interest.

Received: 5 June 2020 Accepted: 11 January 2021

Published online: 22 January 2021

\section{References}

1. Cuadrado E, Michailidou I, van Bodegraven EJ, Jansen MH, Sluijs JA, Geerts $D$, et al. Phenotypic variation in Aicardi-Goutieres syndrome explained by cell-specific IFN-stimulated gene response and cytokine release. J Immunol. 2015;194(8):3623-33

2. Crow YJ, Chase DS, Lowenstein SJ, Szynkiewicz M, Forte GM, Gornall HL, et al. Characterization of human disease phenotypes associated with mutations in TREX1, RNASEH2A, RNASEH2B, RNASEH2C, SAMHD1, ADAR, and IFIH1. Am J Med Genet A. 2015;167A(2):296-312.

3. Günther C, Kind B, Reijns MAM, Berndt N, Martinez-Bueno M, Wolf C, et al. Defective removal of ribonucleotides from DNA promotes systemic autoimmunity. J Clin Invest. 2015;125(1):413-24.

4. Hinze CH, Holzinger D, Lainka E, Haas JP, Speth F, Kallinich T, et al. Practice and consensus-based strategies in diagnosing and managing systemic 
juvenile idiopathic arthritis in Germany. Pediatr Rheumatol Online J. 2018; 16(1):7.

5. Barut K, Adrovic A, Sahin S, Tarcin G, Tahaoglu G, Koker O, et al. Prognosis, complications and treatment response in systemic juvenile idiopathic arthritis patients: A single-center experience. Int J Rheum Dis. 2019;22(9): 1661-9.

6. Yasin S, Schulert GS. Systemic juvenile idiopathic arthritis and macrophage activation syndrome: update on pathogenesis and treatment. Curr Opin Rheumatol. 2018:30(5):514-20.

7. Goldbach-Mansky R. Current status of understanding the pathogenesis and management of patients with NOMID/CINCA. Curr Rheumatol Rep. 2011; 13(2):123-31.

8. Finetti M, Omenetti A, Federici S, Caorsi R, Gattorno M. Chronic Infantile Neurological Cutaneous and Articular (CINCA) syndrome: a review. Orphanet J Rare Dis. 2016:11(1):167.

9. Zheng S, Lee PY, Wang J, Wang S, Huang Q, Huang Y, et al. Interstitial lung disease and psoriasis in a Child with Aicardi-Goutières syndrome. Front Immunol. 2020;11:985.

10. Komaki R, Ueda T, Tsuji Y, Miyawaki T, Kusuhara S, Hara S, et al. Retinal vasculopathy with cerebral leukoencephalopathy carrying TREX1 mutation diagnosed by the intracranial calcification: a case report. Rinsho Shinkeigaku. 2018;58(2):111-7.

11. Bocci V, Pacini A, Muscettola M, Paulesu L, Pessina GP, Santiano M, et al. Renal filtration, absorption and catabolism of human alpha interferon. J Interferon Res. 1981;1(3):347-52.

12. Henrickson $\mathrm{M}$, Wang $\mathrm{H}$. Tocilizumab reverses cerebral vasculopathy in a patient with homozygous SAMHD1 mutation. Clin Rheumatol. 2017;36(6): 1445-51.

13. Tonduti D, Fazzi E, Badolato R, Orcesi S. Novel and emerging treatments for Aicardi-Goutieres syndrome. Expert Rev Clin Immunol. 2020;16(2):189-98.

14. de Jesus AA, Brehm A, VanTries R, Pillet P, Parentelli AS, Montealegre SG, et al. Novel proteasome assembly chaperone mutations in PSMG2/PAC2 cause the autoinflammatory interferonopathy CANDLE/PRAAS4. J Allergy Clin Immunol. 2019;143(5):1939-43.

15. Melki I, Rose Y, Uggenti C, Van Eyck L, Fremond ML, Kitabayashi N, et al. Disease-associated mutations identify a novel region in human STING necessary for the control of type I interferon signaling. J Allergy Clin Immunol. 2017;140(2):543-52.

16. Sanchez G, Reinhardt A, Ramsey S, Wittkowski H, Hashkes PJ, Berkun Y, et al. JAK1/2 inhibition with baricitinib in the treatment of autoinflammatory interferonopathies. J Clin Invest. 2018;128(7):3041-52.

17. Kothur K, Bandodkar S, Chu S, Wienholt L, Johnson A, Barclay P, et al. An open-label trial of JAK $1 / 2$ blockade in progressive IFIH1-associated neuroinflammation. Neurology. 2018;90(6):289-91.

18. Meesilpavikkai K, Dik WA, Schrijver B, van Helden-Meeuwsen CG, Versnel MA, van Hagen PM, et al. Efficacy of Baricitinib in the Treatment of Chilblains Associated With Aicardi-Goutieres Syndrome, a Type Interferonopathy. Arthritis Rheumatol. 2019;71(5):829-31.

19. Parra-Izquierdo I, Castanos-Mollor I, Lopez J, Gomez C, San RJ, Sanchez CM, et al. Calcification induced by type I interferon in human Aortic valve interstitial cells is larger in males and blunted by a janus kinase inhibitor. Arterioscler Thromb Vasc Biol. 2018;38(9):2148-59.

20. Tungler V, Konig N, Gunther C, Engel K, Fiehn C, Smitka M, et al. Response to: 'JAK inhibition in STING-associated interferonopathy' by Crow. Ann Rheum Dis. 2016;75(12):e76.

21. Crow YJ, Shetty J, Livingston JH. Treatments in Aicardi-Goutieres syndrome. Dev Med Child Neurol. 2020;62(1):42-7.

\section{Publisher's Note}

Springer Nature remains neutral with regard to jurisdictional claims in published maps and institutional affiliations.

Ready to submit your research? Choose BMC and benefit from:

- fast, convenient online submission

- thorough peer review by experienced researchers in your field

- rapid publication on acceptance

- support for research data, including large and complex data types

- gold Open Access which fosters wider collaboration and increased citations

- maximum visibility for your research: over $100 \mathrm{M}$ website views per year

At $\mathrm{BMC}$, research is always in progress.

Learn more biomedcentral.com/submissions 